\title{
ON A FAMILY OF INTEGRAL OPERATORS OF HANKEL TYPE
}

\author{
CHRISTOPH UEBERSOHN
}

\begin{abstract}
In this paper we perform an explicit diagonalization of Hankel integral operators $K^{(0)}, K^{(1)}, K^{(2)}, \ldots$ It turns out that each of these operators has a simple purely absolutely continuous spectrum filling in the interval $[-1,1]$. This generalizes a result of Kostrykin and Makarov (2008).
\end{abstract}

\section{INTRODUCTION AND THE MAIN THEOREM}

We denote by $\mathbb{S}^{1}=\{z \in \mathbb{C}:|z|=1\}$ and $H^{2}$ the unit circle and the Hardy space on the circle, respectively. Here and for the rest of the paper we use the following representation of a Hankel operator on $H^{2}$ with symbol $\varphi \in L^{\infty}\left(\mathbb{S}^{1}\right)$ :

$$
S_{\varphi}=\left.P J M_{\varphi}\right|_{H^{2}}
$$

where $P: L^{2}\left(\mathbb{S}^{1}\right) \rightarrow L^{2}\left(\mathbb{S}^{1}\right)$ is the orthogonal projection onto the closed subspace $H^{2}$ of $L^{2}\left(\mathbb{S}^{1}\right), J: L^{2}\left(\mathbb{S}^{1}\right) \rightarrow L^{2}\left(\mathbb{S}^{1}\right)$ denotes the flip operator, $(J f)(z)=f(\bar{z})$, and $M_{\varphi}: L^{2}\left(\mathbb{S}^{1}\right) \rightarrow L^{2}\left(\mathbb{S}^{1}\right)$ is the multiplication operator defined by $M_{\varphi} f=\varphi \cdot f$.

In his 1953 paper [3] Mark Krein considered two bounded integral operators $A_{j}$, $j=0,1$, on $L^{2}(0, \infty)$ with kernels

$$
a_{0}(x, y)=\left\{\begin{array}{ll}
\sinh (x) \mathrm{e}^{-y} & \text { if } x \leq y \\
\sinh (y) \mathrm{e}^{-x} & \text { if } x \geq y
\end{array} \text { and } a_{1}(x, y)=\left\{\begin{array}{ll}
\cosh (x) \mathrm{e}^{-y} & \text { if } x \leq y \\
\cosh (y) \mathrm{e}^{-x} & \text { if } x \geq y
\end{array} .\right.\right.
$$

The operators $A_{j}, j=0,1$, are the resolvents of the one-dimensional Dirichlet $(j=0)$ and Neumann $(j=1)$ Laplacians $-\mathrm{d}^{2} / \mathrm{d} x^{2}$ at the spectral point -1 , respectively. Krein showed that the difference of the spectral projections for the operators $A_{0}$ and $A_{1}$ is given by

$$
K_{\mu}=E_{(-\infty, \mu)}\left(A_{1}\right)-E_{(-\infty, \mu)}\left(A_{0}\right), \quad 0<\mu<1,
$$

where the kernel function of the integral operator $K_{\mu}$ is of the form $k_{\mu}(x+y)$. More precisely,

$$
k_{\mu}(x)=\frac{2}{\pi} \frac{\sin (\sqrt{\lambda(\mu)} x)}{x} \quad \text { with } \lambda(\mu)=\frac{1}{\mu}-1 .
$$

In [2] Vadim Kostrykin and Konstantin A. Makarov deduced from this that $K_{\mu}$ is unitarily equivalent to $K_{1 / 2}$ with kernel $k_{1 / 2}(x+y)=\frac{2}{\pi} \frac{\sin (x+y)}{x+y}$, and $K_{1 / 2}$ is unitarily equivalent to the Hankel operator $S_{z \phi}$ on $H^{2}$ where

$$
\phi: \mathbb{S}^{1} \rightarrow \mathbb{R}, \quad \phi(z)=2 \cdot \mathbb{1}_{\overline{\mathbb{H}_{r}} \cap \mathbb{S}^{1}}(z),
$$

and $\mathbb{H}_{r}=\{z \in \mathbb{C}: \operatorname{Re}(z)>0\}$ denotes the right half plane. By $\mathbb{1}_{\overline{\mathbb{H}_{r}} \cap \mathbb{S}^{1}}$ we denote the characteristic function of $\overline{\mathbb{H}}_{r} \cap \mathbb{S}^{1}=\left\{z \in \mathbb{S}^{1}: \operatorname{Re}(z) \geq 0\right\}$.

Date: November 7, 2018.

2010 Mathematics Subject Classification. Primary 47B35; Secondary 47A10.

Key words and phrases. Hankel operator, integral operator, spectrum, diagonalization. 
In the present paper we consider a "natural" generalization of this operator $S_{z \phi}$, namely the family of operators $S_{z \phi}, S_{z^{2} \phi}, S_{z^{3} \phi}, \ldots$ As we will show in Theorem 2.6. these Hankel operators on $H^{2}$ are unitarily equivalent to the Hankel integral operators $K^{(0)}, K^{(1)}, K^{(2)}, \ldots$ on $L^{2}(0, \infty)$ with kernels $(0, \infty) \times(0, \infty) \rightarrow \mathbb{R}$, $(x, y) \mapsto k^{(\ell)}(x+y)$. These kernels are determined by the functions

$$
\begin{aligned}
k^{(0)}(x) & :=\frac{2}{\pi} \frac{\sin (x)}{x} \\
k^{(\ell)}(x) & :=\frac{1}{\pi} \frac{1}{2^{\ell-1}} \sum_{n=0}^{2 \ell}(-1)^{n}\left(\begin{array}{c}
2 \ell \\
n
\end{array}\right) \frac{\mathrm{d}^{n}}{\mathrm{~d} x^{n}}\left(\left(\mathrm{e}^{-|w|} p_{\ell}(|w|)\right) *\left(\frac{\sin (w)}{w}\right)\right)(x),
\end{aligned}
$$

defined even on the whole of $\mathbb{R}$, where $\sin (0) / 0:=1$,

$$
p_{\ell}(w)=\sum_{j=0}^{\ell-1} \frac{1}{2^{j}}\left(\begin{array}{c}
\ell+j-1 \\
\ell-1
\end{array}\right) \frac{1}{(\ell-j-1) !} w^{\ell-j-1}, \quad w \in \mathbb{R}
$$

and $\ell \in \mathbb{N}:=\{1,2,3, \ldots\}$. Here and for the rest of the paper we denote by $\left(\begin{array}{l}N \\ n\end{array}\right)=$ $\frac{N !}{n !(N-n) !}$ the binomial coefficient " $N$ choose $n$ " if $n$ and $N$ are in $\mathbb{N}_{0}:=\mathbb{N} \cup\{0\}$ such that $n \leq N$. Furthermore, if $f \in L^{p}(\mathbb{R})$ for some $p \in[1, \infty]$ and $g \in L^{1}(\mathbb{R})$, we denote by $f * g$ the convolution of $f$ and $g$,

$$
(f * g)(y):=(f(w) * g(w))(y):=\int_{-\infty}^{\infty} f(y-x) g(x) \mathrm{d} x, \quad y \in \mathbb{R} .
$$

In Theorem 1.1 we will give a full description of the spectrum of each $K^{(0)}$, $K^{(1)}, K^{(2)}, \ldots$

Theorem 1.1. Let $p \leq 1 / 2$ be a real number. Define the functions $\rho_{p}:(0, \infty) \rightarrow$ $(0, \infty)$ and $h:(0, \infty) \rightarrow(0, \infty)$ by

$\rho_{p}(\lambda)=\frac{1}{2 \pi^{2}} \sinh (2 \pi \sqrt{\lambda})\left|\Gamma\left(\frac{1}{2}-p-\mathrm{i} \sqrt{\lambda}\right)\right|^{2} \quad$ and $\quad h(\lambda)=\frac{\pi}{\cosh (\pi \sqrt{\lambda})}$,

respectively, where $\Gamma$ denotes the Gamma function. For all $m \in \mathbb{N}_{0}$ the following results hold true:

(1) The operator $K^{(2 m)}$ is unitarily equivalent to

$M_{\frac{(-1) m}{\pi} h} \oplus M_{\frac{(-1)^{m+1}}{\pi} h}$ on $L^{2}\left((0, \infty) ; \rho_{\frac{1}{2}-m}(\lambda) \mathrm{d} \lambda\right) \oplus L^{2}\left((0, \infty) ; \rho_{-\frac{1}{2}-m}(\lambda) \mathrm{d} \lambda\right)$ and has a simple purely absolutely continuous spectrum filling in the interval $[-1,1]$.

(2) The operator $K^{(2 m+1)}$ is unitarily equivalent to

$M_{\frac{(-1)^{m+1}}{\pi} h} \oplus M_{\frac{(-1)^{m}}{\pi} h}$ on $L^{2}\left((0, \infty) ; \rho_{-\frac{1}{2}-m}(\lambda) \mathrm{d} \lambda\right) \oplus L^{2}\left((0, \infty) ; \rho_{-\frac{1}{2}-m}(\lambda) \mathrm{d} \lambda\right)$ and has a simple purely absolutely continuous spectrum filling in the interval $[-1,1]$.

Furthermore, we will prove some properties of the functions $k^{(1)}, k^{(2)}, k^{(3)}, \ldots$ such as regularity and integrability, and we will study the behaviour of $k^{(\ell)}(x)$ as $|x| \rightarrow \infty$, see Theorem 3.4 and Corollary 3.5. In Theorem 4.5 we present an "explicit" formula for $k^{(\ell)}(x)$ if $\ell \in \mathbb{N}$ and $x>0$, i. e., a formula which neither contains integrals nor convolutions. From this representation of the functions 
$k^{(1)}, k^{(2)}, k^{(3)}, \ldots$ it is immediate that each function $k^{(\ell)}$ is real analytic on $(0, \infty)$, see Corollary 4.6

We know only a few examples of Hankel operators which can be explicitly diagonalized, see Yafaev [7]. From this point of view, it would be interesting to consider $K^{(0)}, K^{(1)}, K^{(2)}, \ldots$ even if we had not known that the operators $K^{(1)}, K^{(2)}, \ldots$ can be regarded as a "natural" generalization of the Hankel operator of Krein's example. Unfortunately, Yafaev's commutator method for the diagonalization of Hankel operators which is described in [7] is not applicable to our problem.

\section{Proof of The MAIN THEOREM}

On the basis of Theorem 2.6 below, we will slightly generalize the proof of Theorem 1 by Kostrykin and Makarov [2] to develop a proof of Theorem 1.1 .

In order to derive Theorem 2.6, we first need to prove some auxiliary results.

We denote by $H^{2}(\mathbb{R})$ the Hardy space on the line. A Hankel operator on $H^{2}(\mathbb{R})$ with symbol $\psi \in L^{\infty}(\mathbb{R})$ acts as follows:

$$
S_{\psi}=\left.Q R M_{\psi}\right|_{H^{2}(\mathbb{R})}
$$

where $Q: L^{2}(\mathbb{R}) \rightarrow L^{2}(\mathbb{R})$ is the orthogonal projection onto the closed subspace $H^{2}(\mathbb{R})$ of $L^{2}(\mathbb{R}), R: L^{2}(\mathbb{R}) \rightarrow L^{2}(\mathbb{R})$ denotes the reflection operator, $(R f)(x)=f(-x)$, and $M_{\psi}: L^{2}(\mathbb{R}) \rightarrow L^{2}(\mathbb{R})$ is the multiplication operator defined by $M_{\psi} f=\psi \cdot f$.

We use the following definition of the Fourier transform: if $f \in L^{1}(\mathbb{R}) \cap L^{2}(\mathbb{R})$ then

Define

$$
(\mathscr{F} f)(x)=\frac{1}{\sqrt{2 \pi}} \int_{-\infty}^{\infty} f(t) \mathrm{e}^{-\mathrm{i} t x} \mathrm{~d} t, \quad x \in \mathbb{R} .
$$

and

$$
\psi: \mathbb{R} \rightarrow \mathbb{R}, \quad \psi(t)=2 \cdot \mathbb{1}_{[-1,1]}(t),
$$

$$
\phi: \mathbb{S}^{1} \rightarrow \mathbb{R}, \quad \phi(z)=2 \cdot \mathbb{1}_{\overline{\mathbb{H}_{r}} \cap \mathbb{S}^{1}}(z)=\left\{\begin{array}{ll}
\psi\left(\mathrm{i} \frac{1-z}{1+z}\right) & \text { if } z \neq-1 \\
0 & \text { if } z=-1
\end{array} .\right.
$$

Lemma 2.1. Let $\ell \in \mathbb{N}_{0}$. Then

$$
\frac{1}{2 \pi} \int_{-\infty}^{\infty} t^{\ell} \psi(t) \mathrm{e}^{-\mathrm{i} t x} \mathrm{~d} t=\sqrt{\frac{2}{\pi}}\left(\mathscr{F}\left\{t^{\ell} \mathbb{1}_{[-1,1]}(t)\right\}\right)(x)=\frac{2}{\pi} \mathrm{i}^{\ell} \frac{\mathrm{d}^{\ell}}{\mathrm{d} x^{\ell}}\left\{\frac{\sin (x)}{x}\right\}
$$

holds true for all $x \in \mathbb{R}$.

Proof. This is straightforward.

Let $\ell \in \mathbb{N}_{0}$. Since

$$
\psi_{\ell}(t):=\left(\frac{1+\mathrm{i} t}{1-\mathrm{i} t}\right)^{\ell} \phi\left(\frac{1+\mathrm{i} t}{1-\mathrm{i} t}\right)=\left(\frac{1+\mathrm{i} t}{1-\mathrm{i} t}\right)^{\ell} \psi(t)
$$

for all $t \in \mathbb{R}$ we know by Power [5], p. 14, that the Hankel operator $S_{\psi_{\ell}}$ on $H^{2}(\mathbb{R})$ is unitarily equivalent to the Hankel operator $S_{z^{\ell+1} \phi}$ on $H^{2}$.

We will compute the Fourier transform of $t \mapsto\left(\frac{1+\mathrm{i} t}{1-\mathrm{i} t}\right)^{\ell} \psi(t)$ for all $\ell \in \mathbb{N}_{0}$. Define $\xi: \mathbb{R} \rightarrow \mathbb{R}, t \mapsto \frac{1}{1+t^{2}}$, and $\tilde{\psi}_{\ell}: \mathbb{R} \rightarrow \mathbb{C}, t \mapsto(1+\mathrm{i} t)^{2 \ell} \cdot \psi(t)$. Since $\frac{1+\mathrm{i} t}{1-\mathrm{i} t}=\frac{(1+\mathrm{i} t)^{2}}{1+t^{2}}$ we have to determine the Fourier transform of $\xi^{\ell} \cdot \tilde{\psi}_{\ell}$. 
We start by computing $\mathscr{F} \tilde{\psi}_{\ell}$. Obviously, $\tilde{\psi}_{\ell} \in L^{1}(\mathbb{R}) \cap L^{2}(\mathbb{R})$ for all $\ell \in \mathbb{N}_{0}$. Now the binomial theorem, the linearity of the Fourier transform, and Lemma 2.1 imply the following representation for $\mathscr{F} \tilde{\psi}_{\ell}$ :

Proposition 2.2. Let $\ell \in \mathbb{N}_{0}$. Then

$$
\left(\mathscr{F} \tilde{\psi}_{\ell}\right)(w)=\sqrt{\frac{8}{\pi}} \sum_{n=0}^{2 \ell}\left(\begin{array}{c}
2 \ell \\
n
\end{array}\right)(-1)^{n} \frac{\mathrm{d}^{n}}{\mathrm{~d} w^{n}} \frac{\sin (w)}{w}, \quad w \in \mathbb{R},
$$

holds true. The function $z \mapsto \frac{\sin (z)}{z}$ is entire; if we restrict it to $\mathbb{R}$ then the function $z \mapsto \frac{\sin (z)}{z}$ and all of its derivatives of arbitrary order are bounded.

Proof. It remains to show that the function $z \mapsto \frac{\sin (z)}{z}=\sum_{n=0}^{\infty}(-1)^{n} \frac{z^{2 n}}{(2 n+1) !}$ possesses the properties claimed above. It follows from the Cauchy-Hadamard theorem and Stirling's approximation that the function $z \mapsto \frac{\sin (z)}{z}$ is entire.

Let $n \in \mathbb{N}_{0}$. Leibniz' rule implies that

$$
\frac{\mathrm{d}^{n}}{\mathrm{~d} x^{n}} \frac{\sin (x)}{x}=\sum_{j=0}^{n}\left(\begin{array}{l}
n \\
j
\end{array}\right)(-1)^{j} j ! x^{-j-1} \frac{\mathrm{d}^{n-j}}{\mathrm{~d} x^{n-j}} \sin (x) \rightarrow 0
$$

as $|x| \rightarrow \infty$ where $x$ is real. Therefore, Proposition 2.2 is proved.

Theorem 2.3. For each $\ell \in \mathbb{N}$ the Fourier transform of $\xi^{\ell}$ is given by

$$
\begin{gathered}
\left(\mathscr{F}\left\{\xi^{\ell}\right\}\right)(w)=\frac{1}{\sqrt{2 \pi}} \cdot \frac{\pi}{2^{\ell-1}} \cdot \mathrm{e}^{-|w|} p_{\ell}(|w|), \quad w \in \mathbb{R}, \text { where } \\
p_{\ell}(w)=\sum_{j=0}^{\ell-1} \frac{1}{2^{j}}\left(\begin{array}{c}
\ell+j-1 \\
\ell-1
\end{array}\right) \frac{1}{(\ell-j-1) !} w^{\ell-j-1}, \quad w \in \mathbb{R} .
\end{gathered}
$$

Remark (to Theorem 2.3). There is a more general result in "Tabellen zur Fourier Transformation" [4] by Oberhettinger; according to p. 202 in [4] one has

$$
\int_{-\infty}^{\infty} \frac{\mathrm{e}^{\mathrm{i} x y}}{\left\{a^{2}+(x \pm b)^{2}\right\}^{\nu}} \mathrm{d} x=2 \mathrm{e}^{\mp \mathrm{i} b y} \frac{\sqrt{\pi}}{\Gamma(\nu)}\left(\frac{|y|}{2 a}\right)^{\nu-1 / 2} K_{\nu-1 / 2}(a|y|), \operatorname{Re}(\nu)>0,
$$

where $\Gamma$ denotes the Gamma function and

$$
\begin{aligned}
& K_{\nu}(z)=\frac{\pi}{2} \frac{I_{-\nu}(z)-I_{\nu}(z)}{\sin (\pi \nu)}, \quad I_{\nu}(z)=\mathrm{e}^{-\mathrm{i} \pi \nu / 2} J_{\nu}\left(z \mathrm{e}^{\mathrm{i} \pi / 2}\right), \\
& J_{\nu}(z)=\sum_{n=0}^{\infty} \frac{(-1)^{n}(z / 2)^{\nu+2 n}}{n ! \Gamma(\nu+n+1)} .
\end{aligned}
$$

In case that $a=1, b=0$, and $\nu=\ell \in \mathbb{N}$, we are in the situation of Theorem 2.3.

We will need the following

Lemma 2.4. Let $\ell \in \mathbb{N}_{0}$. Then

$$
\int_{-\infty}^{\infty}|x|^{\ell} \mathrm{e}^{-|x|} \mathrm{e}^{-|y-x|} \mathrm{d} x=\frac{\mathrm{e}^{-|y|}}{\ell+1}\left\{|y|^{\ell+1}+\sum_{j=0}^{\ell-1} \frac{(\ell+1) !}{(\ell-j) !} \frac{|y|^{\ell-j}}{2^{1+j}}+\frac{(\ell+1) !}{2^{\ell}}\right\}
$$

holds true for all $y \in \mathbb{R}$. 
Proof. In this proof we denote by $\mathrm{H}$ the Heaviside function.

Let $\ell \in \mathbb{N}_{0}, y \in \mathbb{R} \backslash\{0\}$. By elementary computations it follows that

$$
\begin{aligned}
I_{1} & :=\int_{0}^{\infty} x^{\ell} \mathrm{e}^{-x} \mathrm{e}^{-y+x} \mathbb{1}_{\{x<y\}}(x) \mathrm{d} x=\mathrm{e}^{-y} \frac{y^{\ell+1}}{\ell+1} \cdot \mathrm{H}(y), \\
I_{2} & :=\int_{0}^{\infty} x^{\ell} \mathrm{e}^{-x} \mathrm{e}^{y-x} \mathbb{1}_{\{x>y\}}(x) \mathrm{d} x \\
& =\mathrm{e}^{-|y|}\left\{\frac{\ell !}{2^{\ell+1}} \cdot \mathrm{H}(-y)+\frac{1}{2^{\ell+1}} \sum_{j=0}^{\ell}(2 y)^{\ell-j} \frac{\ell !}{(\ell-j) !} \cdot \mathrm{H}(y)\right\}, \\
I_{3} & :=\int_{-\infty}^{0}(-x)^{\ell} \mathrm{e}^{x} \mathrm{e}^{-y+x} \mathbb{1}_{\{x<y\}}(x) \mathrm{d} x \\
& =\mathrm{e}^{-|y|}\left\{\frac{\ell !}{2^{\ell+1}} \cdot \mathrm{H}(y)+\frac{1}{2^{\ell+1}} \sum_{j=0}^{\ell}(2 y)^{\ell-j} \frac{\ell !}{(\ell-j) !} \cdot \mathrm{H}(-y)\right\}, \\
I_{4} & :=\int_{-\infty}^{0}(-x)^{\ell} \mathrm{e}^{x} \mathrm{e}^{y-x} \mathbb{1}_{\{x>y\}}(x) \mathrm{d} x=\mathrm{e}^{y} \frac{(-y)^{\ell+1}}{\ell+1} \cdot \mathrm{H}(-y) .
\end{aligned}
$$

Altogether, we get that

$$
\begin{aligned}
& \int_{-\infty}^{\infty}|x|^{\ell} \mathrm{e}^{-|x|} \mathrm{e}^{-|y-x|} \mathrm{d} x=I_{1}+I_{2}+I_{3}+I_{4} \\
& =\frac{\mathrm{e}^{-|y|}}{\ell+1}\left\{|y|^{\ell+1}+\sum_{j=0}^{\ell-1} 2^{-1-j} \cdot \frac{(\ell+1) !}{(\ell-j) !} \cdot|y|^{\ell-j}+\frac{(\ell+1) !}{2^{\ell}}\right\} .
\end{aligned}
$$

Since $\int_{-\infty}^{\infty}|x|^{\ell} \mathrm{e}^{-|x|} \mathrm{e}^{-|0-x|} \mathrm{d} x=2 \int_{0}^{\infty} x^{\ell} \mathrm{e}^{-2 x} \mathrm{~d} x=\frac{\ell !}{2^{\ell}}$ the proof is complete.

We can now prove Theorem 2.3 .

Proof of Theorem 2.3. Obviously, $\xi^{\ell} \in L^{1}(\mathbb{R}) \cap L^{2}(\mathbb{R})$ for each $\ell \in \mathbb{N}$. We prove Theorem 2.3 by induction on $\ell \in \mathbb{N}$. For $\ell=1$ we use the inverse operator $\mathscr{F}^{-1}$ to show that

Integration by parts easily leads to

$$
(\mathscr{F} \xi)(w)=\sqrt{\frac{\pi}{2}} \cdot \mathrm{e}^{-|w|}, \quad w \in \mathbb{R} .
$$

$$
\left(\mathscr{F}^{-1}\left\{\sqrt{\frac{\pi}{2}} \mathrm{e}^{-|w|}\right\}\right)(t)=\int_{0}^{\infty} \mathrm{e}^{-w} \cos (t w) \mathrm{d} w=\frac{1}{1+t^{2}}, \quad t \in \mathbb{R} .
$$

Suppose that the claim holds up to $\ell$. We have to show that it still holds for $\ell+1$. Taken together, the well-known behaviour of the Fourier transform of the convolution of two functions in $L^{1}(\mathbb{R}) \cap L^{2}(\mathbb{R})$, the induction hypothesis, Lemma 2.4, and a comparison of coefficients yield 


$$
\begin{aligned}
\left(\mathscr{F}\left\{\xi^{\ell+1}\right\}\right)(w)=\frac{1}{\sqrt{2 \pi}}\left(\left(\mathscr{F}\left\{\xi^{\ell}\right\}\right) *(\mathscr{F} \xi)\right)(w) \\
=\frac{1}{\sqrt{2 \pi}} \frac{\pi}{2^{\ell}} \sum_{j=0}^{\ell-1} \frac{1}{2^{j}}\left(\begin{array}{c}
\ell+j-1 \\
\ell-1
\end{array}\right) \frac{1}{(\ell-j-1) !}\left(\left(\mathrm{e}^{-|x|}|x|^{\ell-j-1}\right) *\left(\mathrm{e}^{-|x|}\right)\right)(w) \\
=\frac{1}{\sqrt{2 \pi}} \frac{\pi}{2^{\ell}} \mathrm{e}^{-|w|} \sum_{j=0}^{\ell-1} \frac{1}{2^{j}}\left(\begin{array}{c}
\ell+j-1 \\
\ell-1
\end{array}\right) \frac{1}{(\ell-j) !} \\
\qquad\left\{|w|^{\ell-j}+\sum_{m=0}^{\ell-j-2} 2^{-1-m} \frac{(\ell-j) !}{(\ell-1-j-m) !}|w|^{\ell-1-j-m}+\frac{(\ell-j) !}{2^{\ell-j-1}}\right\} \\
=\frac{\mathrm{e}^{-|w|}}{\sqrt{2 \pi}} \frac{\pi}{2^{\ell}}\left\{\frac{1}{2^{\ell-1}} \sum_{j=0}^{\ell-1}\left(\begin{array}{c}
\ell+j-1 \\
\ell-1
\end{array}\right)+\sum_{\beta=1}^{\ell} \frac{1}{2^{\ell-\beta}} \frac{1}{\beta !} \sum_{\alpha=0}^{\ell-\beta}\left(\begin{array}{c}
\ell+\alpha-1 \\
\ell-1
\end{array}\right)|w|^{\beta}\right\} \\
=\frac{1}{\sqrt{2 \pi}} \frac{\pi}{2^{\ell}} \mathrm{e}^{-|w|}\left\{\frac{1}{2^{\ell-1}}\left(\begin{array}{c}
2 \ell-1 \\
\ell
\end{array}\right)+\sum_{\beta=1}^{\ell} \frac{1}{2^{\ell-\beta}} \frac{1}{\beta !}\left(\begin{array}{c}
2 \ell-\beta \\
\ell
\end{array}\right)|w|^{\beta}\right\} \\
=\frac{1}{\sqrt{2 \pi}} \frac{\pi}{2^{\ell}} \mathrm{e}^{-|w|} \sum_{j=0}^{\ell} \frac{1}{2^{j}}\left(\begin{array}{c}
\ell+j \\
\ell
\end{array}\right) \frac{1}{(\ell-j) !}|w|^{\ell-j}, \quad w \in \mathbb{R} ;
\end{aligned}
$$

note that $\frac{1}{2^{\ell}}\left(\begin{array}{c}2 \ell \\ \ell\end{array}\right)=\frac{1}{2^{\ell}}\left\{\left(\begin{array}{c}2 \ell-1 \\ \ell-1\end{array}\right)+\left(\begin{array}{c}2 \ell-1 \\ \ell\end{array}\right)\right\}=\frac{1}{2^{\ell-1}}\left(\begin{array}{c}2 \ell-1 \\ \ell\end{array}\right)$. This finishes the proof of Theorem 2.3 .

Now Proposition 2.2 and Theorem 2.3 lead to

\section{Corollary 2.5. For the functions}

$$
k^{(\ell)}: \mathbb{R} \rightarrow \mathbb{R}, \quad x \mapsto k^{(\ell)}(x)=\frac{1}{2 \pi} \int_{-\infty}^{\infty} \psi_{\ell}(t) \mathrm{e}^{-\mathrm{i} t x} \mathrm{~d} t, \quad \ell \in \mathbb{N},
$$

one has

$$
\begin{aligned}
k^{(\ell)}(x) & =\frac{1}{\sqrt{2 \pi}}\left(\mathscr{F}\left\{\xi^{\ell} \cdot \tilde{\psi}_{\ell}\right\}\right)(x) \\
& =\frac{1}{\pi} \frac{1}{2^{\ell-1}} \sum_{n=0}^{2 \ell}(-1)^{n}\left(\begin{array}{c}
2 \ell \\
n
\end{array}\right) \frac{\mathrm{d}^{n}}{\mathrm{~d} x^{n}}\left(\left(\mathrm{e}^{-|w|} p_{\ell}(|w|)\right) *\left(\frac{\sin (w)}{w}\right)\right)(x) .
\end{aligned}
$$

We denote by $\mathscr{D}(0, \infty)$ the set of all test functions on $(0, \infty)$, i. e. the set of all infinitely differentiable functions on $(0, \infty)$ with compact support.

Theorem 2.6. Let $\ell \in \mathbb{N}_{0}$. Consider the integral operator $K^{(\ell)}: L^{2}(0, \infty) \rightarrow$ $L^{2}(0, \infty)$ with kernel function $k^{(\ell)}(x+y)$,

$$
\left(K^{(\ell)} f\right)(x)=\int_{0}^{\infty} k^{(\ell)}(x+y) f(y) \mathrm{d} y, \quad f \in \mathscr{D}(0, \infty), \quad x>0,
$$

on the dense subspace $\mathscr{D}(0, \infty) \subset L^{2}(0, \infty)$. Recall that $k^{(0)}(x+y)=\frac{2}{\pi} \frac{\sin (x+y)}{x+y}$. Then $K^{(\ell)}$ is a bounded self-adjoint Hankel operator which is unitarily equivalent to the Hankel operator $S_{\psi_{\ell}}$ with symbol $\psi_{\ell}$ on $H^{2}(\mathbb{R})$, and $S_{\psi_{\ell}}$ is unitarily equivalent to the Hankel operator $S_{z^{\ell+1} \phi}$ on $H^{2}$, for all $\ell \in \mathbb{N}_{0}$. Here $\phi$ denotes the function which is defined as in (2.1). 
Proof. Let $\ell \in \mathbb{N}$. According to Theorem 3.4 below, the function $k^{(\ell)}$ is continuous on $(0, \infty)$, and one has $k^{(\ell)}(x)=\mathcal{O}\left(x^{-1}\right)$ both as $x \rightarrow 0+$ and as $x \rightarrow \infty$. Obviously, the function $\psi_{\ell}=\xi^{\ell} \cdot \tilde{\psi}_{\ell}$ defined as in 2.2 is in $L^{1}(\mathbb{R}) \cap L^{2}(\mathbb{R}) \cap$ $L^{\infty}(\mathbb{R})$, and according to Corollary 2.5 one has

$$
\frac{1}{\sqrt{2 \pi}} \mathscr{F} \psi_{\ell}=\frac{1}{\sqrt{2 \pi}} \mathscr{F}\left\{\xi^{\ell} \cdot \tilde{\psi}_{\ell}\right\}=k^{(\ell)}
$$

on the whole of $\mathbb{R}$. It follows that the integral operator $K^{(\ell)}$ with kernel function $k^{(\ell)}(x+y)$ is the uniquely defined bounded Hankel operator on $L^{2}(0, \infty)$ which is defined by

$$
\left(K^{(\ell)} f\right)(x)=\int_{0}^{\infty} k^{(\ell)}(x+y) f(y) \mathrm{d} y, \quad f \in \mathscr{D}(0, \infty), \quad x>0,
$$

on the dense subspace $\mathscr{D}(0, \infty) \subset L^{2}(0, \infty)$. According to Corollary 2.5 the function $k^{(\ell)}$ is real-valued so $\overline{k^{(\ell)}(y+x)}=k^{(\ell)}(x+y)$ for all $x, y>0$. Therefore, $K^{(\ell)}$ is self-adjoint. Now $K^{(\ell)}$ is unitarily equivalent to $S_{\psi_{\ell}}$ with symbol $\psi_{\ell}$ on $H^{2}(\mathbb{R})$, and $S_{\psi_{\ell}}$ is unitarily equivalent to $S_{z^{\ell+1} \phi}$ on $H^{2}$ by Power [5], p. 14 .

In case that $\ell=0$ the proof runs analogously.

We denote by $\ell_{+}^{2}$ the Hilbert space of all complex square-summable one-sided sequences $x=\left(x_{0}, x_{1}, \ldots\right)$.

Lemma 2.7. Let $\phi$ be defined as in (2.1) and

$$
c_{k}=\frac{1}{2 \pi} \int_{0}^{2 \pi} \mathrm{e}^{\mathrm{i} k \theta} \phi\left(\mathrm{e}^{\mathrm{i} \theta}\right) \mathrm{d} \theta=\frac{2}{\pi k} \sin (\pi k / 2), \quad k \in \mathbb{N} .
$$

Then for each $x \in \ell_{+}^{2}$

$$
\left(S_{z^{\ell+1} \phi} x\right)_{n}=\sum_{k=0}^{\infty} c_{k+\ell+n+1} x_{k}, \quad \ell \in \mathbb{N}_{0}
$$

holds true.

Proof. This is straightforward.

Let $\mathcal{P}_{+}$and $\mathcal{P}_{-}$be the orthogonal projections in $\ell_{+}^{2}$ onto $\mathcal{L}_{+}$and $\mathcal{L}_{-}$, respectively, where

$$
\begin{gathered}
\mathcal{L}_{+}=\left\{x \in \ell_{+}^{2}: x_{2 k+1}=0 \text { for all } k \in \mathbb{N}_{0}\right\}, \\
\mathcal{L}_{-}=\left\{x \in \ell_{+}^{2}: x_{2 k}=0 \text { for all } k \in \mathbb{N}_{0}\right\} .
\end{gathered}
$$

Let $p \leq 1 / 2$ be a real number. Consider the operators $H_{p}: \ell_{+}^{2} \rightarrow \ell_{+}^{2}$ and $\widetilde{H}_{p}: \ell_{+}^{2} \rightarrow \ell_{+}^{2}$ defined by

$$
\left(H_{p} x\right)_{n}=\sum_{k=0}^{\infty} \frac{x_{k}}{1+k+n-p} \text { and }\left(\widetilde{H}_{p} x\right)_{n}=\sum_{k=0}^{\infty} \frac{(-1)^{k+n}}{1+k+n-p} x_{k}, \quad x \in \ell_{+}^{2},
$$

respectively. Both the operators $H_{p}$ and $\widetilde{H}_{p}$ are bounded and self-adjoint, and one has $V \widetilde{H}_{p} V=H_{p}$ where

$$
V: \ell_{+}^{2} \rightarrow \ell_{+}^{2}, \quad\left(x_{0}, x_{1}, x_{2}, x_{3}, \ldots\right) \mapsto\left(x_{0},-x_{1}, x_{2},-x_{3}, \ldots\right),
$$

is unitary and $V^{-1}=V$. 
First, consider the case when $\ell \in \mathbb{N}_{0}$ is even, i. e. $\ell=2 m, m \in \mathbb{N}_{0}$. With Lemma 2.7, it is easy to compute that

$$
\begin{array}{r}
\left(\mathcal{P}_{+} S_{z^{2 m+1} \phi} \mathcal{P}_{+} x\right)_{2 n}=\frac{1}{\pi} \sum_{k=0}^{\infty} \frac{(-1)^{k+m+n}}{k+m+n+1 / 2} x_{2 k}, \\
\left(\mathcal{P}_{-} S_{z^{2 m+1} \phi} \mathcal{P}_{-} x\right)_{2 n+1}=-\frac{1}{\pi} \sum_{k=0}^{\infty} \frac{(-1)^{k+m+n}}{k+m+n+3 / 2} x_{2 k+1}, \\
\mathcal{P}_{+} S_{z^{2 m+1} \phi} \mathcal{P}_{-}=0=\mathcal{P}_{-} S_{z^{2 m+1} \phi} \mathcal{P}_{+} .
\end{array}
$$

Define the operators

$$
\mathcal{U}_{+}: \ell_{+}^{2} \rightarrow \mathcal{U}_{+}\left(\ell_{+}^{2}\right), \quad x=\left(x_{0}, x_{1}, x_{2}, \ldots\right) \mapsto\left(x_{0}, 0, x_{1}, 0, x_{2}, 0, \ldots\right),
$$

and

$$
\mathcal{U}_{-}: \ell_{+}^{2} \rightarrow \mathcal{U}_{-}\left(\ell_{+}^{2}\right), \quad x=\left(x_{0}, x_{1}, x_{2}, \ldots\right) \mapsto\left(0, x_{0}, 0, x_{1}, 0, x_{2}, 0, \ldots\right) .
$$

Both $\mathcal{U}_{+}$and $\mathcal{U}_{-}$are unitary, and it is easy to see that

$$
\begin{aligned}
& \mathcal{P}_{+} S_{z^{2 m+1} \phi} \mathcal{P}_{+} \text {leaves invariant } \mathcal{U}_{+}\left(\ell_{+}^{2}\right), \\
& \mathcal{P}_{-} S_{z^{2 m+1} \phi} \mathcal{P}_{-} \text {leaves invariant } \mathcal{U}_{-}\left(\ell_{+}^{2}\right) .
\end{aligned}
$$

One has

$$
V \circ \mathcal{U}_{ \pm}^{-1} \circ\left(\mathcal{P}_{ \pm} S_{z^{2 m+1} \phi} \mathcal{P}_{ \pm}\right) \circ \mathcal{U}_{ \pm} \circ V= \pm \frac{(-1)^{m}}{\pi} H_{ \pm \frac{1}{2}-m}
$$

where $V$ is defined as in $\sqrt{2.3}$. Since the operator $\mathcal{U}: \ell_{+}^{2} \rightarrow \mathcal{U}_{+}\left(\ell_{+}^{2}\right) \oplus \mathcal{U}_{-}\left(\ell_{+}^{2}\right)$ defined by $\mathcal{U}_{x}=\mathcal{P}_{+} x \oplus \mathcal{P}_{-} x$ is unitary we have shown:

Lemma 2.8. For all $m \in \mathbb{N}_{0}$ the operator $S_{z^{2 m+1} \phi}: \ell_{+}^{2} \rightarrow \ell_{+}^{2}$ is unitarily equivalent to the operator $\left(\frac{(-1)^{m}}{\pi} H_{\frac{1}{2}-m}\right) \oplus\left(\frac{(-1)^{m+1}}{\pi} H_{-\frac{1}{2}-m}\right): \ell_{+}^{2} \oplus \ell_{+}^{2} \rightarrow \ell_{+}^{2} \oplus \ell_{+}^{2}$.

Now consider the case when $\ell \in \mathbb{N}_{0}$ is odd, i. e. $\ell=2 m+1, m \in \mathbb{N}_{0}$. With Lemma 2.7, it is easy to check that

$$
\begin{array}{r}
\mathcal{P}_{+} S_{z^{2 m+2}{ }^{2}} \mathcal{P}_{+}=0=\mathcal{P}_{-} S_{z^{2 m+2} \phi} \mathcal{P}_{-}, \\
\left(\mathcal{P}_{+} S_{z^{2 m+2} \phi} \mathcal{P}_{-} x\right)_{2 n}=-\frac{1}{\pi} \sum_{k=0}^{\infty} \frac{(-1)^{k+m+n}}{k+m+n+3 / 2} x_{2 k+1}, \\
\left(\mathcal{P}_{-} S_{z^{2 m+2} \phi} \mathcal{P}_{+} x\right)_{2 n+1}=-\frac{1}{\pi} \sum_{k=0}^{\infty} \frac{(-1)^{k+m+n}}{k+m+n+3 / 2} x_{2 k}
\end{array}
$$

Let $x \oplus y \in \ell_{+}^{2} \oplus \ell_{+}^{2}$. One has

$$
\begin{aligned}
& {\left[\begin{array}{ll}
V & 0 \\
0 & V
\end{array}\right]\left[\begin{array}{cc}
\mathcal{U}_{+}^{-1} & 0 \\
0 & \mathcal{U}_{-}^{-1}
\end{array}\right]\left[\begin{array}{cc}
0 & \mathcal{P}_{+} S_{z^{2 m+2} \phi} \mathcal{P}_{-} \\
\mathcal{P}_{-} S_{z^{2 m+2} \phi} \mathcal{P}_{+} & 0
\end{array}\right]\left[\begin{array}{cc}
\mathcal{U}_{+} & 0 \\
0 & \mathcal{U}_{-}
\end{array}\right]\left[\begin{array}{ll}
V & 0 \\
0 & V
\end{array}\right]\left[\begin{array}{l}
x \\
y
\end{array}\right]} \\
& =\left[\begin{array}{cc}
0 & \frac{(-1)^{m+1}}{\pi} H_{-\frac{1}{2}-m} \\
\frac{(-1)^{m+1}}{\pi} H_{-\frac{1}{2}-m} & 0
\end{array}\right]\left[\begin{array}{l}
x \\
y
\end{array}\right] .
\end{aligned}
$$

We denote by $I$ the identity operator $\ell_{+}^{2} \rightarrow \ell_{+}^{2}$. Obviously, the operator

$$
\frac{1}{\sqrt{2}}\left[\begin{array}{cc}
I & -I \\
I & I
\end{array}\right]: \ell_{+}^{2} \oplus \ell_{+}^{2} \rightarrow \ell_{+}^{2} \oplus \ell_{+}^{2}
$$


is unitary and its inverse is given by

$$
\frac{1}{\sqrt{2}}\left[\begin{array}{cc}
I & I \\
-I & I
\end{array}\right]: \ell_{+}^{2} \oplus \ell_{+}^{2} \rightarrow \ell_{+}^{2} \oplus \ell_{+}^{2}
$$

Since

$$
\begin{aligned}
& \frac{1}{\sqrt{2}}\left[\begin{array}{cc}
I & I \\
-I & I
\end{array}\right]\left[\begin{array}{cc}
0 & \frac{(-1)^{m+1}}{\pi} H_{-\frac{1}{2}-m} \\
\frac{(-1)^{m+1}}{\pi} H_{-\frac{1}{2}-m} & 0
\end{array}\right] \frac{1}{\sqrt{2}}\left[\begin{array}{cc}
I & -I \\
I & I
\end{array}\right] \\
& =\left[\begin{array}{cc}
\frac{(-1)^{m+1}}{\pi} H_{-\frac{1}{2}-m} & 0 \\
0 & \frac{(-1)^{m}}{\pi} H_{-\frac{1}{2}-m}
\end{array}\right]
\end{aligned}
$$

we have shown:

Lemma 2.9. For all $m \in \mathbb{N}_{0}$ the operator $S_{z^{2 m+2} \phi}: \ell_{+}^{2} \rightarrow \ell_{+}^{2}$ is unitarily equivalent to the operator $\left(\frac{(-1)^{m+1}}{\pi} H_{-\frac{1}{2}-m}\right) \oplus\left(\frac{(-1)^{m}}{\pi} H_{-\frac{1}{2}-m}\right): \ell_{+}^{2} \oplus \ell_{+}^{2} \rightarrow \ell_{+}^{2} \oplus \ell_{+}^{2}$.

Now Theorem 2.6, Lemma 2.8, and Lemma 2.9 allow us to complete the proof of Theorem 1.1 .

Proof of Theorem 1.1. Let $h:(0, \infty) \rightarrow(0, \infty)$ be defined as in Theorem 1.1 Due to Rosenblum [6], Theorem 4 , the operator $H_{p}$ is unitarily equivalent to the multiplication operator $M_{h}$ on $L^{2}\left((0, \infty) ; \rho_{p}(\lambda) \mathrm{d} \lambda\right)$ for all $p \leq 1 / 2$, in particular if $p \in\left\{\frac{1}{2}-m: m \in \mathbb{N}_{0}\right\}$. Therefore, the operators $\frac{1}{\pi} H_{p}$ and $-\frac{1}{\pi} H_{p}$ are unitarily equivalent to the multiplication operators $M_{\tilde{h}}$ and $M_{-\tilde{h}}$ on $L^{2}\left((0, \infty) ; \rho_{p}(\lambda) \mathrm{d} \lambda\right)$, respectively, where $\tilde{h}:=h / \pi$ and $p \in\left\{\frac{1}{2}-m: m \in \mathbb{N}_{0}\right\}$. From this, Theorem 2.6. and from the Lemmas 2.8 and 2.9, all the assertions in Theorem 1.1 follow.

\section{SOME PROPERTIES OF THE FUNCTIONS $k^{(\ell)}$ FOR $\ell \in \mathbb{N}$}

If $\ell=0$ then the function $k^{(0)}: \mathbb{R} \rightarrow \mathbb{R}$ is given by $k^{(0)}(x)=\frac{2}{\pi} \frac{\sin (x)}{x}$; this function is fully understood. In this section, we will prove some properties of $k^{(\ell)}$ for $\ell \in \mathbb{N}$. First we need to prove some lemmas.

Lemma 3.1. Let $\ell \in \mathbb{N}$. Then one has
(1) $\sum_{j=0}^{\ell-1} \frac{\cos ((\ell-j) \pi / 4)}{2^{(\ell+j-2) / 2}}\left(\begin{array}{c}\ell+j-1 \\ \ell-1\end{array}\right)=1$.
(2) $\sum_{n=0}^{\ell}(-1)^{n}\left(\begin{array}{c}2 \ell \\ 2 n\end{array}\right)=\cos (\ell \pi / 2) \cdot 2^{\ell}$.
(3) $\sum_{n=0}^{\ell-1}(-1)^{n+1}\left(\begin{array}{c}2 \ell \\ 2 n+1\end{array}\right)=\sin (-\ell \pi / 2) \cdot 2^{\ell}$. 
Proof. (1) Let $\ell \in \mathbb{N}$. Define $a_{\ell}:=\sum_{j=0}^{\ell-1} \frac{\mathrm{e}^{\mathrm{i} \cdot(\ell-j) \pi / 4}}{2^{(\ell+j) / 2}}\left(\begin{array}{c}\ell+j-1 \\ \ell-1\end{array}\right)$. It is easy to compute

$$
\begin{aligned}
a_{\ell+1} & =\sum_{j=0}^{\ell} \frac{\mathrm{e}^{\mathrm{i} \cdot(\ell+1-j) \pi / 4}}{2^{(\ell+1+j) / 2}}\left(\begin{array}{c}
\ell+j-1 \\
\ell-1
\end{array}\right)+\sum_{j=1}^{\ell} \frac{\mathrm{e}^{\mathrm{i} \cdot(\ell+1-j) \pi / 4}}{2^{(\ell+1+j) / 2}}\left(\begin{array}{c}
\ell+j-1 \\
\ell
\end{array}\right) \\
& =a_{\ell} \cdot \frac{1+\mathrm{i}}{2}+\frac{1+\mathrm{i}}{2^{\ell+1}}\left(\begin{array}{c}
2 \ell-1 \\
\ell-1
\end{array}\right)+a_{\ell+1} \cdot \frac{1-\mathrm{i}}{2}-\frac{1}{2^{\ell+1}}\left(\begin{array}{c}
2 \ell \\
\ell
\end{array}\right)
\end{aligned}
$$

which is equivalent to

$$
a_{\ell+1}=a_{\ell}+\frac{\mathrm{i}}{2^{\ell+1}}\left(\begin{array}{c}
2 \ell \\
\ell
\end{array}\right) .
$$

From this it follows that

$$
\frac{1}{2}=\operatorname{Re}\left(a_{1}\right)=\ldots=\operatorname{Re}\left(a_{\ell}\right)=\sum_{j=0}^{\ell-1} \frac{\cos ((\ell-j) \pi / 4)}{2^{(\ell+j) / 2}}\left(\begin{array}{c}
\ell+j-1 \\
\ell-1
\end{array}\right) .
$$

(2) If $\ell \in \mathbb{N}$ then

$$
\sum_{n=0}^{\ell}(-1)^{n}\left(\begin{array}{l}
2 \ell \\
2 n
\end{array}\right)=\operatorname{Re}\left\{(1+\mathrm{i})^{2 \ell}\right\}=\cos (\ell \pi / 2) \cdot 2^{\ell} .
$$

(3) If $\ell \in \mathbb{N}$ then

$$
\sum_{n=0}^{\ell-1}(-1)^{n+1}\left(\begin{array}{c}
2 \ell \\
2 n+1
\end{array}\right)=-\operatorname{Im}\left\{(1+\mathrm{i})^{2 \ell}\right\}=\sin (-\ell \pi / 2) \cdot 2^{\ell} .
$$

Lemma 3.2. Let $m \in \mathbb{N}_{0}$. Then one has

(1) $\int_{-\infty}^{\infty} \mathrm{e}^{-|y|}|y|^{m} \sin (x-y) \mathrm{d} y=\frac{m !}{2^{(m-1) / 2}} \cos ((m+1) \pi / 4) \cdot \sin (x)$ for all $x \in \mathbb{R}$.

(2) $\int_{-\infty}^{\infty} \mathrm{e}^{-|y|}|y|^{m} \cos (x-y) \mathrm{d} y=\frac{m !}{2^{(m-1) / 2}} \cos ((m+1) \pi / 4) \cdot \cos (x)$ for all $x \in \mathbb{R}$.

Proof. Let $m \in \mathbb{N}_{0}$.

(1) Let $x \in \mathbb{R}$. It is straightforward to compute

$$
\begin{aligned}
\int_{-\infty}^{\infty} \mathrm{e}^{-|y|}|y|^{m} \sin (x-y) \mathrm{d} y & =2 \sin (x) \int_{0}^{\infty} \mathrm{e}^{-y} y^{m} \cos (y) \mathrm{d} y \\
& =\sin (x)\left\{\frac{m !}{2^{(m-1) / 2}} \cos ((m+1) \pi / 4)\right\} .
\end{aligned}
$$

(2) Put $\tilde{x}=x+\pi / 2, x \in \mathbb{R}$. Since $\sin (\tilde{x}-y)=\cos (x-y)$ for every $y \in \mathbb{R}$ the claim follows from the first part of the lemma. 
Lemma 3.3. Let $\ell \in \mathbb{N}$. Then

$$
\begin{aligned}
& \frac{1}{\pi} \frac{1}{2^{\ell-1}} \sum_{n=0}^{2 \ell}(-1)^{n}\left(\begin{array}{c}
2 \ell \\
n
\end{array}\right)\left(\left(\mathrm{e}^{-|w|} p_{\ell}(|w|)\right) *\left(\frac{\mathrm{d}^{n}}{\mathrm{~d} w^{n}} \sin (w)\right)\right)(x) \\
& =\frac{2}{\pi} \cdot \sin (x-\ell \pi / 2)
\end{aligned}
$$

holds true for each $x \in \mathbb{R}$.

Proof. Let $\ell \in \mathbb{N}$. It follows from Lemma 3.2 and Lemma 3.1 that

$$
\begin{aligned}
& \frac{1}{\pi} \frac{1}{2^{\ell-1}} \sum_{n=0}^{2 \ell}(-1)^{n}\left(\begin{array}{c}
2 \ell \\
n
\end{array}\right)\left(\left(\mathrm{e}^{-|w|} p_{\ell}(|w|)\right) *\left(\frac{\mathrm{d}^{n}}{\mathrm{~d} w^{n}} \sin (w)\right)\right)(x) \\
& =\frac{\sin (x)}{\pi \cdot 2^{\ell-1}}\left[\sum_{j=0}^{\ell-1} \frac{\cos ((\ell-j) \pi / 4)}{2^{(\ell+j-2) / 2}}\left(\begin{array}{c}
\ell+j-1 \\
\ell-1
\end{array}\right)\right] \cdot\left[\sum_{\tilde{n}=0}^{\ell}(-1)^{\tilde{n}}\left(\begin{array}{c}
2 \ell \\
2 \tilde{n}
\end{array}\right)\right] \\
& +\frac{\cos (x)}{\pi \cdot 2^{\ell-1}}\left[\sum_{j=0}^{\ell-1} \frac{\cos ((\ell-j) \pi / 4)}{2^{(\ell+j-2) / 2}}\left(\begin{array}{c}
\ell+j-1 \\
\ell-1
\end{array}\right)\right] \cdot\left[\sum_{\tilde{n}=0}^{\ell-1}(-1)^{\tilde{n}+1}\left(\begin{array}{c}
2 \ell \\
2 \tilde{n}+1
\end{array}\right)\right] \\
& =\frac{2}{\pi} \cdot \sin (x-\ell \pi / 2), \quad x \in \mathbb{R} .
\end{aligned}
$$

We denote by $\mathscr{C}^{\infty}(\mathbb{R})$ the set of all infinitely differentiable functions on $\mathbb{R}$.

Theorem 3.4. Let $\ell \in \mathbb{N}$. Then one has:

(1) $k^{(\ell)} \in \mathscr{C}^{\infty}(\mathbb{R}) \cap L^{2}(\mathbb{R}) \cap L^{\infty}(\mathbb{R})$. All derivatives of arbitrary order of $k^{(\ell)}$ are in $\mathscr{C}^{\infty}(\mathbb{R}) \cap L^{\infty}(\mathbb{R})$. In particular, the limit $\lim _{x \rightarrow 0+} k^{(\ell)}(x)$ exists in $\mathbb{R}$.

(2) $k^{(\ell)}(x)=\mathcal{O}\left(x^{-1}\right)$ as $|x| \rightarrow \infty$, i.e. $\limsup \left|x \cdot k^{(\ell)}(x)\right| \leq C<\infty$ for some constant $C>0$.

(3) $\lim _{|x| \rightarrow \infty}\left|x \cdot k^{(\ell)}(x)-\frac{2}{\pi} \cdot \sin (x-\ell \pi / 2)\right|=0$.

Proof. Let $\ell \in \mathbb{N}$.

(1) We just show the boundedness. All the other assertions are obvious. Let $m \in \mathbb{N}_{0}$. Define the following positive constants:

$C^{\prime \prime}:=\max \left\{\sup _{w \in \mathbb{R}}\left|\frac{\mathrm{d}^{j}}{\mathrm{~d} w^{j}} \frac{\sin (w)}{w}\right|: j \in\{0,1, \ldots, 2 \ell+m\}\right\}$

$C^{\prime}:=\max \left\{\frac{1}{\pi} \frac{\left(\begin{array}{c}\ell+j-1 \\ \ell-1\end{array}\right)}{2^{j+\ell-1}} \frac{\left(\begin{array}{c}2 \ell \\ n\end{array}\right)}{(\ell-j-1) !}: j \in\{0, \ldots, \ell-1\}, n \in\{0, \ldots, 2 \ell\}\right\}$,

$C:=C^{\prime} \cdot C^{\prime \prime}$. 
One has

$$
\begin{aligned}
\left|\frac{\mathrm{d}^{m}}{\mathrm{~d} x^{m}} k^{(\ell)}(x)\right| & \leq C \cdot \sum_{j=0}^{\ell-1} \sum_{n=0}^{2 \ell} \int_{-\infty}^{\infty} \mathrm{e}^{-|y|}|y|^{\ell-j-1} \mathrm{~d} y \\
& =2(2 \ell+1) C \cdot \sum_{j=0}^{\ell-1}(\ell-j-1) !
\end{aligned}
$$

for all $x \in \mathbb{R}$. Since this constant is finite and does not depend on $x$ we have shown that each derivative of arbitrary order of $k^{(\ell)}$ is in $L^{\infty}(\mathbb{R})$.

(2) This is immediate by the third part of the theorem.

(3) Let $n \in \mathbb{N}_{0}$. The functions

$$
F_{2 n}: z \mapsto z \cdot \frac{\mathrm{d}^{2 n}}{\mathrm{~d} z^{2 n}} \frac{\sin (z)}{z}-(-1)^{n} \sin (z)
$$

and

$$
G_{2 n+1}: z \mapsto z \cdot \frac{\mathrm{d}^{2 n+1}}{\mathrm{~d} z^{2 n+1}} \frac{\sin (z)}{z}-(-1)^{n} \cos (z)
$$

are entire. Furthermore, Leibniz' rule implies that

$$
x \cdot \frac{\mathrm{d}^{2 n}}{\mathrm{~d} x^{2 n}} \frac{\sin (x)}{x}-(-1)^{n} \sin (x)=\sum_{m=1}^{2 n}\left(\begin{array}{c}
2 n \\
m
\end{array}\right)(-1)^{m} m ! x^{-m} \frac{\mathrm{d}^{2 n-m}}{\mathrm{~d} x^{2 n-m}} \sin (x)
$$

and

$$
\begin{aligned}
& x \cdot \frac{\mathrm{d}^{2 n+1}}{\mathrm{~d} x^{2 n+1}} \frac{\sin (x)}{x}-(-1)^{n} \cos (x) \\
& =\sum_{m=1}^{2 n+1}\left(\begin{array}{c}
2 n+1 \\
m
\end{array}\right)(-1)^{m} m ! x^{-m} \frac{\mathrm{d}^{2 n+1-m}}{\mathrm{~d} x^{2 n+1-m}} \sin (x)
\end{aligned}
$$

tend to zero as $|x| \rightarrow \infty$ where $x$ is real. Therefore, $F_{2 n}, G_{2 n+1} \in$ $\mathscr{C}^{\infty}(\mathbb{R}) \cap L^{\infty}(\mathbb{R})$. It is easy to show that the dominated convergence theorem implies

(*)

$$
\left\{\begin{array}{l}
\lim _{|x| \rightarrow \infty} \int_{-\infty}^{\infty} \mathrm{e}^{-|y|} p_{\ell}(|y|) F_{2 n}(x-y) \mathrm{d} y=0 \\
\lim _{|x| \rightarrow \infty} \int_{-\infty}^{\infty} \mathrm{e}^{-|y|} p_{\ell}(|y|) G_{2 n+1}(x-y) \mathrm{d} y=0
\end{array}\right.
$$

for all $\ell \in \mathbb{N}$.

We already know that the function $x \mapsto \frac{\mathrm{d}^{n}}{\mathrm{~d} x^{n}} \frac{\sin (x)}{x}$ is in $\mathscr{C}^{\infty}(\mathbb{R}) \cap$ $L^{\infty}(\mathbb{R})$ and that $\frac{\mathrm{d}^{n}}{\mathrm{~d} x^{n}} \frac{\sin (x)}{x} \rightarrow 0$ for each sequence of real numbers such that $|x| \rightarrow \infty$. It is easy to show that the dominated convergence theorem implies

$$
\lim _{|x| \rightarrow \infty} \int_{-\infty}^{\infty} \mathrm{e}^{-|y|} p_{\ell}(|y|) \cdot y \cdot \frac{\partial^{n}}{\partial x^{n}} \frac{\sin (x-y)}{x-y} \mathrm{~d} y=0
$$

for all $\ell \in \mathbb{N}$. Now Lemma 3.3 , $(*)$, and $(* *)$ yield 


$$
\begin{aligned}
\mid x & \cdot k^{(\ell)}(x)-\frac{2}{\pi} \cdot \sin (x-\ell \pi / 2) \mid \\
= & \mid \frac{1}{\pi} \frac{1}{2^{\ell-1}} \sum_{\tilde{n}=0}^{\ell}\left(\begin{array}{c}
2 \ell \\
2 \tilde{n}
\end{array}\right) \int_{-\infty}^{\infty} \mathrm{e}^{-|y|} p_{\ell}(|y|) F_{2 \tilde{n}}(x-y) \mathrm{d} y \\
& -\frac{1}{\pi} \frac{1}{2^{\ell-1}} \sum_{\tilde{n}=0}^{\ell-1}\left(\begin{array}{c}
2 \ell \\
2 \tilde{n}+1
\end{array}\right) \int_{-\infty}^{\infty} \mathrm{e}^{-|y|} p_{\ell}(|y|) G_{2 \tilde{n}+1}(x-y) \mathrm{d} y \\
& +\frac{1}{\pi} \frac{1}{2^{\ell-1}} \sum_{n=0}^{2 \ell}(-1)^{n}\left(\begin{array}{c}
2 \ell \\
n
\end{array}\right) \int_{-\infty}^{\infty} \mathrm{e}^{-|y|} p_{\ell}(|y|) \cdot y \cdot \frac{\partial^{n}}{\partial x^{n}} \frac{\sin (x-y)}{x-y} \mathrm{~d} y \mid \\
\leq & \frac{1}{\pi} \frac{1}{2^{\ell-1}} \sum_{\tilde{n}=0}^{\ell}\left(\begin{array}{c}
2 \ell \\
2 \tilde{n}
\end{array}\right)\left|\int_{-\infty}^{\infty} \mathrm{e}^{-|y|} p_{\ell}(|y|) F_{2 \tilde{n}}(x-y) \mathrm{d} y\right| \\
& +\frac{1}{\pi} \frac{1}{2^{\ell-1}} \sum_{\tilde{n}=0}^{\ell-1}\left(\begin{array}{c}
2 \ell \\
2 \tilde{n}+1
\end{array}\right)\left|\int_{-\infty}^{\infty} \mathrm{e}^{-|y|} p_{\ell}(|y|) G_{2 \tilde{n}+1}(x-y) \mathrm{d} y\right| \\
& +\frac{1}{\pi} \frac{1}{2^{\ell-1}} \sum_{n=0}^{2 \ell}\left(\begin{array}{c}
2 \ell \\
n
\end{array}\right)\left|\int_{-\infty}^{\infty} \mathrm{e}^{-|y|} p_{\ell}(|y|) \cdot y \cdot \frac{\partial^{n}}{\partial x^{n}} \frac{\sin (x-y)}{x-y} \mathrm{~d} y\right| \\
\rightarrow & 0 \\
& \quad \text { as }|x| \rightarrow \infty, \text { for all } \ell \in \mathbb{N} .
\end{aligned}
$$

\section{Corollary 3.5. Let $\ell \in \mathbb{N}$. Then one has:}

(1) $k^{(\ell)} \in L^{p}(\mathbb{R})$ for all $p \in(1, \infty]$.

(2) $k^{(\ell)} \notin L^{1}(\mathbb{R})$.

Proof. Let $\ell \in \mathbb{N}$.

(1) We already know that $k^{(\ell)} \in L^{\infty}(\mathbb{R})$. If $p \in(1, \infty)$ and $N \in \mathbb{N}$, it is straightforward to compute

$$
\int_{-\infty}^{\infty}\left|k^{(\ell)}(x)\right|^{p} \mathrm{~d} x \leq C_{1}+\frac{2 C_{2}}{p-1} N^{-p+1}<\infty
$$

where $C_{1}:=2 N \cdot\left\|k^{(\ell)}\right\|_{L^{\infty}(\mathbb{R})}^{p}<\infty$ and $C_{2}:=\sup _{x \in \mathbb{R}}\left|x \cdot k^{(\ell)}(x)\right|^{p}<\infty$ by Theorem 3.4

(2) Denote by $B_{\rho}(y) \subset \mathbb{R}$ the open ball of radius $\rho>0$ around a real number $y$.

Put $\varepsilon:=\frac{\pi}{4}>0$. Now it follows from the third part of Theorem 3.4 that

$$
\lim _{n \rightarrow \infty} \frac{k^{(\ell)}\left(x_{n}\right)}{\frac{2}{\pi} \frac{\sin \left(x_{n}-\ell \pi / 2\right)}{x_{n}}}=1 \quad \text { for each sequence }\left(x_{n}\right)_{n \in \mathbb{N}}
$$

in $\Omega:=\mathbb{R} \backslash \bigcup_{m \in \mathbb{Z}} B_{\varepsilon}((m+\ell / 2) \pi)$ such that $\lim _{n \rightarrow \infty}\left|x_{n}\right|=\infty$. Since the function $x \mapsto \frac{k^{(\ell)}(x)}{\frac{2}{\pi} \frac{\sin (x-\ell \pi / 2)}{x}}$ is continuous on $\Omega$ there exists some number 
$N \in \mathbb{N}$ such that $N \geq \ell \pi / 2$ and

$$
\frac{k^{(\ell)}(x)}{\frac{2}{\pi} \frac{\sin (x-\ell \pi / 2)}{x}} \geq 1-\varepsilon>0
$$

for all $x \in[N, \infty) \cap \Omega$. If we put $\widetilde{\Omega}=\mathbb{R} \backslash \bigcup_{m \in \mathbb{Z}} B_{\varepsilon}(m \pi)$, one has

$$
\begin{aligned}
\int_{-\infty}^{\infty}\left|k^{(\ell)}(x)\right| \mathrm{d} x & \geq(1-\varepsilon) \frac{2}{\pi} \int_{\Omega \cap(N, \infty)}\left|\frac{\sin (x-\ell \pi / 2)}{x}\right| \mathrm{d} x \\
& =(1-\varepsilon) \frac{2}{\pi} \int_{\tilde{\Omega} \cap(N+\ell \pi / 2, \infty)}\left|\frac{\sin (\tilde{x}-\ell \pi)}{\tilde{x}-\ell \pi / 2}\right| \mathrm{d} \tilde{x} \\
& \geq(1-\varepsilon) \frac{2}{\pi} \int_{\widetilde{\Omega} \cap(N+\ell \pi / 2, \infty)} \frac{|\sin (\tilde{x})|}{\tilde{x}} \mathrm{~d} \tilde{x}
\end{aligned}
$$

where we substituted $\tilde{x}:=x+\ell \pi / 2$. Since $N \geq \ell \pi / 2$ and $|\sin (x)| \geq \frac{1}{\sqrt{2}}$ for all $x \in \widetilde{\Omega}$ this implies

$$
\begin{aligned}
\int_{-\infty}^{\infty}\left|k^{(\ell)}(x)\right| \mathrm{d} x & \geq(1-\varepsilon) \frac{\sqrt{2}}{\pi} \int_{\widetilde{\Omega} \cap(N \pi+\pi / 4, \infty)} \frac{1}{x} \mathrm{~d} x \\
& =(1-\varepsilon) \frac{\sqrt{2}}{\pi} \sum_{n=N}^{\infty} \int_{n \pi+\pi / 4}^{n \pi+3 \pi / 4} \frac{1}{x} \mathrm{~d} x \\
& \geq(1-\varepsilon) \frac{\sqrt{2}}{2 \pi} \sum_{n=N+1}^{\infty} \frac{1}{n}=\infty .
\end{aligned}
$$

\section{Explicit COMPUTATION OF THE FUnCtions $k^{(\ell)}$ FOR $\ell \in \mathbb{N}$}

In this section, we will compute the functions $k^{(\ell)}$ on $(0, \infty)$. For this we need the exponential integral function $E_{1}$ and the complementary exponential integral function Ein. Recall that Ein : $\mathbb{C} \rightarrow \mathbb{C}$ is entire and can be represented as follows:

$$
\operatorname{Ein}(z)=\int_{0}^{1} \frac{1-\mathrm{e}^{-t z}}{t} \mathrm{~d} t, \quad z \in \mathbb{C} .
$$

It is well-known that the exponential integral function $E_{1}$ is holomorphic on $\mathbb{C}^{-}:=$ $\mathbb{C} \backslash(-\infty, 0]$ and can be represented on $\overline{\mathbb{H}_{r}} \backslash\{0\}$ as follows:

$$
E_{1}(z)=\int_{1}^{\infty} \frac{\mathrm{e}^{-t z}}{t} \mathrm{~d} t, \quad z \in \overline{\mathbb{H}_{r}} \backslash\{0\} .
$$

Furthermore, if $z$ is in $\mathbb{C}^{-}$then

$$
E_{1}(z)=\operatorname{Ein}(z)-\log (z)-\gamma
$$

where $\log$ is the principal value of the complex logarithm and $\gamma$ denotes Euler's constant.

First we will prove some lemmas. 
According to [1] , p. 217, formula (14), we know the following fact:

Lemma 4.1. Let a be in $\mathbb{C}, \operatorname{Re}(a)>0$, and $n \in \mathbb{N}_{0}$. Then the following formula holds true for all $x>0$ :

$$
\int_{0}^{\infty} y^{n} \mathrm{e}^{-a y} \frac{1}{x+y} \mathrm{~d} y=(-1)^{n} x^{n} \mathrm{e}^{a x} E_{1}(a x)+\sum_{r=1}^{n}(-1)^{n-r}(r-1) ! a^{-r} x^{n-r} .
$$

We will also need

Lemma 4.2. The following formulas hold true:

(1) For all $x>0$,

$\int_{0}^{\infty} \mathrm{e}^{-y} \frac{\sin (x-y)}{x-y} \mathrm{~d} y=\pi \mathrm{e}^{-x}+\frac{\mathrm{i}}{2} \mathrm{e}^{-x} \cdot\left\{E_{1}(-x-\mathrm{i} x)-E_{1}(-x+\mathrm{i} x)\right\}$.

(2) Let $m \in \mathbb{N}$. If $r \in\{1, \ldots, m\}$ then

$$
\sum_{j=r}^{m}(-1)^{j}\left(\begin{array}{c}
m \\
j
\end{array}\right) \frac{(j-1) !}{(j-r) !}=(-1)^{r}(r-1) !
$$

(3) If $m \in \mathbb{N}_{0}$ then

$$
\begin{aligned}
& \int_{0}^{\infty} \mathrm{e}^{-y} y^{m} \frac{\sin (x-y)}{x-y} \mathrm{~d} y \\
& =\left(\pi \mathrm{e}^{-x}+\frac{\mathrm{i}}{2} \mathrm{e}^{-x} \cdot\left\{E_{1}(-x-\mathrm{i} x)-E_{1}(-x+\mathrm{i} x)\right\}\right) \cdot x^{m} \\
& \quad+\sum_{r=1}^{m} \frac{(r-1) !}{2^{r / 2}} x^{m-r} \sin \left(\frac{r \pi}{4}-x\right), \quad x>0 .
\end{aligned}
$$

Proof. (1) From (4.1) and 4.3) it follows that

$$
\begin{aligned}
& \int_{-1}^{1} \frac{\mathrm{e}^{-y(x-\mathrm{i} x)}-\mathrm{e}^{-y(x+\mathrm{i} x)}}{y} \mathrm{~d} y \\
& =-\operatorname{Ein}(x-\mathrm{i} x)+\operatorname{Ein}(-x+\mathrm{i} x)+\operatorname{Ein}(x+\mathrm{i} x)-\operatorname{Ein}(-x-\mathrm{i} x) \\
& =-E_{1}(x-\mathrm{i} x)+E_{1}(-x+\mathrm{i} x)+E_{1}(x+\mathrm{i} x)-E_{1}(-x-\mathrm{i} x)+2 \pi \mathrm{i}, \quad x>0 .
\end{aligned}
$$

If we substitute $\hat{y}:=-1+y / x$ and then apply 4.2) we get that

$$
\begin{aligned}
\int_{0}^{\infty} & \mathrm{e}^{-y} \frac{\sin (x-y)}{x-y} \mathrm{~d} y=\frac{\mathrm{e}^{-x}}{2 \mathrm{i}} \int_{-1}^{\infty}\left(-\mathrm{e}^{-\hat{y}(x+\mathrm{i} x)}+\mathrm{e}^{-\hat{y}(x-\mathrm{i} x)}\right) \frac{1}{\hat{y}} \mathrm{~d} \hat{y} \\
& =\frac{\mathrm{e}^{-x}}{2 \mathrm{i}}\left\{E_{1}(x-\mathrm{i} x)-E_{1}(x+\mathrm{i} x)+\int_{-1}^{1} \frac{\mathrm{e}^{-\hat{y}(x-\mathrm{i} x)}-\mathrm{e}^{-\hat{y}(x+\mathrm{i} x)}}{\hat{y}} \mathrm{~d} \hat{y}\right\} \\
& =\pi \mathrm{e}^{-x}+\frac{\mathrm{i}}{2} \mathrm{e}^{-x} \cdot\left\{E_{1}(-x-\mathrm{i} x)-E_{1}(-x+\mathrm{i} x)\right\}, \quad x>0 .
\end{aligned}
$$

(2) It is not hard to show

$$
\sum_{j=r}^{m}(-1)^{j}\left(\begin{array}{c}
m \\
j
\end{array}\right)\left(\begin{array}{l}
j-1 \\
r-1
\end{array}\right)=(-1)^{r} \quad \text { for all } r \in\{1, \ldots, m\}
$$

by induction on $m \in \mathbb{N}$. 
(3) If $m=0$ then the assertion follows immediately from (1).

Now let $m$ be in $\mathbb{N}$. Let $a$ be in $\mathbb{C}$ such that $\operatorname{Re}(a)>0$. Then the substitution $r:=1+k$, a comparison of coefficients, and the second part of this lemma imply

$$
\begin{aligned}
& x^{m} \sum_{j=1}^{m}\left(\begin{array}{c}
m \\
j
\end{array}\right)(-1)^{j} \sum_{k=0}^{j-1}\left(\begin{array}{c}
j-1 \\
k
\end{array}\right)(-1)^{k} x^{-1-k} \int_{0}^{\infty} \mathrm{e}^{-a y} y^{k} \mathrm{~d} y \\
& =x^{m} \sum_{j=1}^{m}\left(\begin{array}{c}
m \\
j
\end{array}\right)(-1)^{j} \sum_{k=0}^{j-1}\left(\begin{array}{c}
j-1 \\
k
\end{array}\right)(-1)^{k} \frac{k !}{a^{1+k}} x^{-1-k} \\
& =x^{m} \sum_{r=1}^{m} a^{-r} x^{-r}(-1)^{r+1} \sum_{j=r}^{m}(-1)^{j}\left(\begin{array}{c}
m \\
j
\end{array}\right) \frac{(j-1) !}{(j-r) !} \\
& =-\sum_{r=1}^{m} a^{-r}(r-1) ! x^{m-r}, \quad x>0 .
\end{aligned}
$$

From the binomial theorem, $(*)$, and the first part of this lemma it follows that

$$
\begin{aligned}
& \int_{0}^{\infty} \mathrm{e}^{-y}\{(y-x)+x\}^{m} \frac{\sin (x-y)}{x-y} \mathrm{~d} y \\
&=x^{m} \int_{0}^{\infty} \mathrm{e}^{-y} \frac{\sin (x-y)}{x-y} \mathrm{~d} y \\
& \quad+\frac{\mathrm{e}^{\mathrm{i} x}}{2 \mathrm{i}} x^{m} \sum_{j=1}^{m}\left(\begin{array}{c}
m \\
j
\end{array}\right)(-1)^{j} \sum_{k=0}^{j-1}\left(\begin{array}{c}
j-1 \\
k
\end{array}\right) \frac{(-1)^{k}}{x^{k+1}} \int_{0}^{\infty} \mathrm{e}^{-(1+\mathrm{i}) y} y^{k} \mathrm{~d} y \\
&-\frac{\mathrm{e}^{-\mathrm{i} x}}{2 \mathrm{i}} x^{m} \sum_{j=1}^{m}\left(\begin{array}{c}
m \\
j
\end{array}\right)(-1)^{j} \sum_{k=0}^{j-1}\left(\begin{array}{c}
j-1 \\
k
\end{array}\right) \frac{(-1)^{k}}{x^{k+1}} \int_{0}^{\infty} \mathrm{e}^{-(1-\mathrm{i}) y} y^{k} \mathrm{~d} y \\
&=\left(\pi \mathrm{e}^{-x}+\frac{\mathrm{i}}{2} \mathrm{e}^{-x} \cdot\left\{E_{1}(-x-\mathrm{i} x)-E_{1}(-x+\mathrm{i} x)\right\}\right) \cdot x^{m} \\
&-\frac{\mathrm{e}^{\mathrm{i} x}}{2 \mathrm{i}} \sum_{r=1}^{m}(1+\mathrm{i})^{-r}(r-1) ! x^{m-r}+\frac{\mathrm{e}^{-\mathrm{i} x}}{2 \mathrm{i}} \sum_{r=1}^{m}(1-\mathrm{i})^{-r}(r-1) ! x^{m-r} \\
&=\left(\pi \mathrm{e}^{-x}+\frac{\mathrm{i}}{2} \mathrm{e}^{-x} \cdot\left\{E_{1}(-x-\mathrm{i} x)-E_{1}(-x+\mathrm{i} x)\right\}\right) \cdot x^{m} \\
&+\sum_{r=1}^{m} \frac{(r-1) !}{2^{r / 2}} x^{m-r} \sin \left(\frac{r \pi}{4}-x\right), \quad x>0,
\end{aligned}
$$

as was to be shown. 
Lemma 4.3. Let $m, n \in \mathbb{N}_{0}$ such that $m \geq n$. Then one has:

(1) For all $x>0$,

$$
\begin{aligned}
& P_{m, n}^{-}(x):=\int_{0}^{\infty} \mathrm{e}^{-y} y^{m} \frac{\partial^{n}}{\partial x^{n}} \frac{\sin (x-y)}{x-y} \mathrm{~d} y \\
& =\left(\pi \mathrm{e}^{-x}+\frac{\mathrm{i}}{2} \mathrm{e}^{-x}\left\{E_{1}(-x-\mathrm{i} x)-E_{1}(-x+\mathrm{i} x)\right\}\right) \sum_{j=0}^{n}\left(\begin{array}{l}
n \\
j
\end{array}\right) \frac{(-1)^{n-j} m !}{(m-j) !} x^{m-j} \\
& \quad+\sum_{j=0}^{n}\left(\begin{array}{l}
n \\
j
\end{array}\right)(-1)^{n-j} \frac{m !}{(m-j) !} \sum_{r=1}^{m-j} \frac{(r-1) !}{2^{r / 2}} \sin \left(\frac{r \pi}{4}-x\right) x^{m-j-r} .
\end{aligned}
$$

(2) For all $x>0$,

$$
\begin{aligned}
& P_{m, n}^{+}(x):=\int_{0}^{\infty} \mathrm{e}^{-y} y^{m} \frac{\partial^{n}}{\partial x^{n}} \frac{\sin (x+y)}{x+y} \mathrm{~d} y \\
& =(-1)^{m} \frac{\mathrm{i}}{2} \mathrm{e}^{x}\left\{E_{1}(x+\mathrm{i} x)-E_{1}(x-\mathrm{i} x)\right\} \sum_{j=0}^{n}\left(\begin{array}{l}
n \\
j
\end{array}\right) \frac{m !}{(m-j) !} x^{m-j} \\
& \quad+\sum_{j=0}^{n}\left(\begin{array}{l}
n \\
j
\end{array}\right) \frac{m !}{(m-j) !} \sum_{r=1}^{m-j}(-1)^{m-r} \frac{(r-1) !}{2^{r / 2}} \sin \left(\frac{r \pi}{4}+x\right) x^{m-j-r} .
\end{aligned}
$$

Proof. (1) It is straightforward to compute

$$
\begin{aligned}
& \int_{0}^{\infty} \mathrm{e}^{-y} y^{m} \frac{\partial^{n}}{\partial x^{n}} \frac{\sin (x-y)}{x-y} \mathrm{~d} y=\int_{0}^{\infty} \frac{\partial^{n}}{\partial y^{n}}\left\{\mathrm{e}^{-y} y^{m}\right\} \frac{\sin (x-y)}{x-y} \mathrm{~d} y \\
& =\sum_{j=0}^{n}\left(\begin{array}{c}
n \\
j
\end{array}\right)(-1)^{n-j} \frac{m !}{(m-j) !} \int_{0}^{\infty} \mathrm{e}^{-y} y^{m-j} \frac{\sin (x-y)}{x-y} \mathrm{~d} y, \quad x>0,
\end{aligned}
$$

where we used integration by parts and Leibniz' rule. Now the claim follows from the third part of Lemma 4.2 .

(2) This is proved analogously to the first part of this lemma; just use Lemma 4.1 instead of Lemma 4.2.

Lemma 4.4. Let $m \in \mathbb{N}$ and $n \in \mathbb{N}_{0}$. Then the following results hold true:

(1) For all $x>0$,

$$
\int_{0}^{\infty} \mathrm{e}^{-y} \frac{\partial^{n}}{\partial y^{n}} \frac{\sin (x-y)}{x-y} \mathrm{~d} y=\sum_{b=0}^{n-1}(-1)^{n+b} \frac{\mathrm{d}^{n-1-b}}{\mathrm{~d} x^{n-1-b}} \frac{\sin (x)}{x}+\int_{0}^{\infty} \mathrm{e}^{-y} \frac{\sin (x-y)}{x-y} \mathrm{~d} y .
$$

(2) For all $x>0$,

$$
\int_{0}^{\infty} \mathrm{e}^{-y} \frac{\partial^{n}}{\partial y^{n}} \frac{\sin (x+y)}{x+y} \mathrm{~d} y=-\sum_{b=0}^{n-1} \frac{\mathrm{d}^{n-1-b}}{\mathrm{~d} x^{n-1-b}} \frac{\sin (x)}{x}+\int_{0}^{\infty} \mathrm{e}^{-y} \frac{\sin (x+y)}{x+y} \mathrm{~d} y
$$


(3) If $m<n$ then

$$
\begin{aligned}
& Q_{m, n}^{-}(x):=\int_{0}^{\infty} \mathrm{e}^{-y} y^{m} \frac{\partial^{n}}{\partial x^{n}} \frac{\sin (x-y)}{x-y} \mathrm{~d} y \\
& =(-1)^{n-m-1} \sum_{s=0}^{n-m-1}(-1)^{s} \frac{(n-s-1) !}{(n-m-s-1) !} \frac{\mathrm{d}^{s}}{\mathrm{~d} x^{s}} \frac{\sin (x)}{x} \\
& \quad+\left(\pi \mathrm{e}^{-x}+\frac{\mathrm{i}}{2} \mathrm{e}^{-x}\left\{E_{1}(-x-\mathrm{i} x)-E_{1}(\mathrm{i} x-x)\right\}\right) \sum_{j=0}^{m}\left(\begin{array}{c}
n \\
j
\end{array}\right) \frac{(-1)^{n-j} m !}{(m-j) !} x^{m-j} \\
& \quad+\sum_{j=0}^{m}\left(\begin{array}{c}
n \\
j
\end{array}\right)(-1)^{n-j} \frac{m !}{(m-j) !} \sum_{r=1}^{m-j} \frac{(r-1) !}{2^{r / 2}} \sin \left(\frac{r \pi}{4}-x\right) x^{m-j-r}, \quad x>0 .
\end{aligned}
$$

(4) If $m<n$ then

$$
\begin{aligned}
& Q_{m, n}^{+}(x):=\int_{0}^{\infty} \mathrm{e}^{-y} y^{m} \frac{\partial^{n}}{\partial x^{n}} \frac{\sin (x+y)}{x+y} \mathrm{~d} y \\
& =(-1)^{m+1} \sum_{s=0}^{n-m-1} \frac{(n-s-1) !}{(n-m-s-1) !} \frac{\mathrm{d}^{s}}{\mathrm{~d} x^{s}} \frac{\sin (x)}{x} \\
& \quad+(-1)^{m} \frac{\mathrm{i}}{2} \mathrm{e}^{x}\left\{E_{1}(x+\mathrm{i} x)-E_{1}(x-\mathrm{i} x)\right\} \sum_{j=0}^{m}\left(\begin{array}{c}
n \\
j
\end{array}\right) \frac{m !}{(m-j) !} x^{m-j} \\
& \quad+\sum_{j=0}^{m}\left(\begin{array}{c}
n \\
j
\end{array}\right) \frac{m !}{(m-j) !} \sum_{r=1}^{m-j}(-1)^{m-r} \frac{(r-1) !}{2^{r / 2}} \sin \left(\frac{r \pi}{4}+x\right) x^{m-j-r}, \quad x>0 .
\end{aligned}
$$

Proof. (1) This is easily proved by induction on $n \in \mathbb{N}_{0}$.

(2) This is easily proved by induction on $n \in \mathbb{N}_{0}$, too.

(3) It follows analogously to the proof of the first part of Lemma 4.3 that

$$
\begin{aligned}
& \int_{0}^{\infty} \mathrm{e}^{-y} y^{m} \frac{\partial^{n}}{\partial x^{n}} \frac{\sin (x-y)}{x-y} \mathrm{~d} y \\
& =\mathrm{e}^{-x}\left(\pi+\frac{\mathrm{i}}{2}\left\{E_{1}(-x-\mathrm{i} x)-E_{1}(\mathrm{i} x-x)\right\}\right) \sum_{j=0}^{m}\left(\begin{array}{l}
n \\
j
\end{array}\right) \frac{(-1)^{n-j} m !}{(m-j) !} x^{m-j} \\
& \quad+\sum_{j=0}^{m}\left(\begin{array}{c}
n \\
j
\end{array}\right)(-1)^{n-j} \frac{m !}{(m-j) !} \sum_{r=1}^{m-j} \frac{(r-1) !}{2^{r / 2}} \sin \left(\frac{r \pi}{4}-x\right) x^{m-j-r}
\end{aligned}
$$

(*) $\quad+$ boundary terms

for all $x>0$. Since

$\int_{0}^{\infty} \mathrm{e}^{-y} \frac{\partial^{j}}{\partial y^{j}} \frac{\sin (x-y)}{x-y} \mathrm{~d} y=\int_{0}^{\infty} \mathrm{e}^{-y} \frac{\partial^{j-1}}{\partial y^{j-1}} \frac{\sin (x-y)}{x-y} \mathrm{~d} y+$ boundary term and

$$
\frac{\mathrm{d}^{m+j}}{\mathrm{~d} y^{m+j}}\left\{\mathrm{e}^{-y} y^{m}\right\}=m !(-1)^{j}\left(\begin{array}{c}
m+j \\
m
\end{array}\right) \mathrm{e}^{-y}+\text { remainder }
$$


for all $j \in \mathbb{N}$ it follows with (1) that the boundary terms in $(*)$ are given by

$$
\begin{aligned}
& m ! \sum_{a=0}^{n-m-1}\left(\begin{array}{c}
m+a-1 \\
m-1
\end{array}\right)(-1)^{a} \sum_{b=0}^{n-m-a-1}(-1)^{b} \frac{\mathrm{d}^{n-m-a-1-b}}{\mathrm{~d} x^{n-m-a-1-b}} \frac{\sin (x)}{x} \\
& =m ! \sum_{r=0}^{n-m-1}(-1)^{r} \frac{\mathrm{d}^{n-m-r-1}}{\mathrm{~d} x^{n-m-r-1}} \frac{\sin (x)}{x} \sum_{a=0}^{r}\left(\begin{array}{c}
m+a-1 \\
m-1
\end{array}\right) \\
& =(-1)^{n-m-1} \sum_{s=0}^{n-m-1}(-1)^{s} \frac{(n-s-1) !}{(n-m-s-1) !} \frac{\mathrm{d}^{s}}{\mathrm{~d} x^{s}} \frac{\sin (x)}{x}, \quad x>0,
\end{aligned}
$$

where we substituted $r:=a+b$, compared coefficients, and put $s:=$ $n-m-r-1$. Now the claim follows.

(4) This is proved analogously to the third part of this lemma; just use (2) instead of (1).

Theorem 4.5. Let $\ell \in \mathbb{N}$. Then one has

$$
\begin{aligned}
k^{(\ell)}(x) & \frac{1}{\pi} \frac{1}{2^{2 \ell-2}}\left\{\sum_{m=0}^{\ell-1} \sum_{n=0}^{m}(-1)^{n}\left(\begin{array}{c}
2 \ell \\
n
\end{array}\right) \frac{2^{m}}{m !}\left(\begin{array}{c}
2 \ell-m-2 \\
\ell-1
\end{array}\right)\left[P_{m, n}^{-}(x)+P_{m, n}^{+}(x)\right]\right. \\
& \left.+\sum_{m=0}^{\ell-1} \sum_{n=m+1}^{2 \ell}(-1)^{n}\left(\begin{array}{c}
2 \ell \\
n
\end{array}\right) \frac{2^{m}}{m !}\left(\begin{array}{c}
2 \ell-m-2 \\
\ell-1
\end{array}\right)\left[Q_{m, n}^{-}(x)+Q_{m, n}^{+}(x)\right]\right\}
\end{aligned}
$$

for all $x>0$ where the functions $P_{m, n}^{ \pm}(\cdot)$ and $Q_{m, n}^{ \pm}(\cdot)$ are defined as in Lemma 4.3 and Lemma 4.4 respectively.

Proof. Under the assumptions of this theorem and with the index shift defined by $m:=\ell-j-1$ it is straightforward to prove the formula in Theorem 4.5.

Corollary 4.6. Let $\ell$ be in $\mathbb{N}$. Then the function $k^{(\ell)}$ is real analytic on $(0, \infty)$.

\section{ACKNOWLEDGEMENT}

The present paper is based on the author's Master's thesis "Über eine diskrete Familie von Integraloperatoren". The author would like to thank his supervisor, Vadim Kostrykin, for long and fruitful discussions about Hankel operators and methods of diagonalizing them. Furthermore, the author would like to thank Dmitri Yafaev for a useful question, Manfred Lehn for the simplification of the proof of (2) and (3) in Lemma 3.1, and Hans Heinrich Leithoff for reading the manuscript.

\section{REFERENCES}

[1] A. Erdélyi, W. Magnus, F. Oberhettinger, F. G. Tricomi, Tables of integral transforms, Volume 2, McGraw-Hill, 1954

[2] V. Kostrykin, K. A. Makarov, On Krein's example, Proc. Amer. Math. Soc. 136 (2008), 20672071

[3] M. G. Krein, On the trace formula in perturbation theory, Mat. Sbornik N.S. 33(75) (1953), 597-626 (Russian)

[4] F. Oberhettinger, Tabellen zur Fourier Transformation, Springer, 1957

[5] S. C. Power, Hankel operators on Hilbert space, Pitman, Boston, MA, 1982

[6] M. Rosenblum, On the Hilbert matrix, II, Proc. Amer. Math. Soc. 9 (1958), 581-585 
[7] D. R. Yafaev, A commutator method for the diagonalization of Hankel operators, Funct. Anal. Appl. 44 (2010), no. 4, 295-306

C. UEBERSOHN,

FB 08 - InStitut FÜR MATHEMATIK, JOHANNES GUTENBERG-UniVERSitÄt MAINZ, STAUdinger Weg 9, D-55099 MAINZ, GERMANY

E-mail address: uebersoc@mathematik.uni-mainz.de 